\title{
Science Fiction, Psychologie und Religion
}

\author{
von Alexander Keul
}

In dieser Studie geht es darum, mögliche Zusammenhänge zwischen den populären Kultur-Genres „Science Fiction“ (= SF) und „Fantasy“ und dem menschlichen Unbewußten aus der Sicht von Tiefenpsychologie und Religionswissenschaft zu skizzieren. Ich will versuchen, einige Strukturen pars pro toto quasi journalistisch darzustellen, um jenen Faden weiterzuspinnen, den ich in einer Seminar-Diskussion mit Michael Schmolke (Universität Salzburg) zum Thema „SF-Comics“ am 17. Januar 1985 aufgriff. Es ging damals um die Fragen „Warum expandiert der Sektor SF-Comics auf dem Buch-und Zeitschriftenmarkt so rasch?" und „Woher beziehen die Produzenten des Genres, die SF-Comic-Zeichner, ihre Einfälle?“ Ohne viel nachzudenken entwickelte ich aus dem „Bedürfnisansatz“ (verkürzt etwa: „Was publizistisch Erfolg hat, befriedigt reale Bedürfnisse der Rezipienten") und tiefenpsychologischem Hintergrundwissen die These: „SF-Comics (und andere SF-Produkte, ebenso der Sektor ,Fantasy') sind eine symbolische Darstellung tiefer innerer unbewußter Konflikte und sind in unserer durchrationalisierten, entmythologisierten westlichen Zivilisation letzte irrationale Inseln, technisierte Mythen, eine Art Ersatzreligion im wissenschaftlichen Gewand." Schmolke verwies in seiner Antwort auf die massive Freud-Kritik der letzten Zeit ${ }^{1}$ und fragte sich, wie denn SFComic-Zeichner den Zugang zu diesen - angeblich unbewußten Phantasien der Menschheit fänden. Die folgenden Überlegungen sind die ausführliche Fassung einer Antwort. Was haben die heute vertretenen Schulen der Tiefenpsychologie beziehungsweise auch die Religionswissenschaft zum Themenkomplex „Science Fiction/Fantasy“ zu sagen und wie - durch welche Kommunikationsprozesse - stellt man sich den Weg aus dem Unbewußten in die bildliche oder schriftliche Darstellung im Massenkommunikationsprozeß vor?

\section{Begriffsbestimmungen}

Vor der eigentlichen Auseinandersetzung mit dem Thema erscheint mir eine möglichst genaue Klärung der zentralen Begriffe unbedingt nötig.

Science Fiction: Nach Barmeyer ${ }^{2}$,kann noch keine SF-Definition Anspruch darauf erheben, dem monströsen Gegenstand in seiner ganzen Ausdehnung kritisch-präzise gerecht zu werden“. „In der Bezeichnung Science Fiction treten zwei Begriffe zusammen, die in einer grundsätzlichen Spannung zueinander stehen. Während ,Science' Motive der wissenschaftlichen Rationalität (die sich keineswegs nur auf die Naturwissenschaften bezieht), der technischen Planung, der Nüchternheit und Sachlichkeit signalisiert, verweist ,Fiction' eher auf nicht rationale, emotionale, imaginative Fähigkeiten des Menschen: auf die Phantasie. So heißt die russische Bezeichnung für Science Fiction

Dr. phil. Dr. rer. nat. Alexander Keul kam als gelernter Astronom und Meteorologe mit „auffälligen" Himmelsbeobachtungen (und -beobachtern), in seinem Zweitstudium (Psychologie und Publizistik) mit der Interpretation von Science Fiction in Berührung. Er ist z.Zt. wissenschaftlicher Mitarbeiter am Institut für Psychologie der Universität Salzburg. 
eigentlich sehr viel treffender ,Nautschnaja Fantastika', wissenschaftliche Phantastik (Hervorhebung von mir - A.K.) ${ }^{3}$. Ein australischer Ureinwohner würde nach der Lektüre eines SF- oder Fantasy-Stückes vermutlich sagen: „Das sind Träume (dreaming) des Weißen Mannes.“ Mit „Traumzeit“"(Alcheringa) sprechen die Aborigines denselben irrationalen, unbewußten Bereich an, den Barmeyer Phantasie nennt und noch etwas mehr. „Traumzeit" ist das sprachliche Symbol für ein Kontinuum, für dessen Charakterisierung der westliche Begriffsspalter zahlreiche Bezeichnungen - Phantasie, Glaube, religiöse Erfahrung, Grenzerfahrung, Grenzbereich, Übersinnliches, Unbewußtes, Primärprozeß, Es, andere Wirklichkeit, Trance, Entrückung und ähnliches - verwenden müßte. Es mutet in diesem Zusammenhang fast ironisch an, daß die AlcheringaFiguren alle mundlos (also sprach-los) dargestellt werden ${ }^{4}$.

Fantasy: Dazu meint Barmeyer ${ }^{5}$, OObwohl die SF-Theoretiker fast immer scharf trennen zwischen Science Fiction und der sogenannten ,Fantasy' (einer modernen, der Magie, Märchen- und Sagenmotiven zugewandten phantastischen Literatur, ...) ... “, bestehe dieser Unterschied mehr in den Gehirnen als im Erleben, denn kaum ein SF-Magazin würde Fantasy zurückweisen. Ähnlich argumentiert auch Nagl, der meint: „So ist die Mischung und der Übergang von Science Fiction über Science Fantasy und Heroic Fantasy zu Sword und Sorcery (die Mischung aus Mantel-und Degen-, Schwert- und Zauberei-Genre) stets fließend. ${ }^{\text {“6 }}$ Im folgenden trenne ich daher die beiden Begriffsbildungen durch Schrägstrich, verwende sie aber nebeneinander.

UFO: Ein terminus technicus aus dem Abkürzungslexikon der US-Luftwaffe (US Air Force) der fünfziger Jahre, der über Medienberichte zum Synonym für „Fliegende Untertasse“ oder „außerirdisches Raumschiff“ (mit grünen Männchen) wurde. Ursprünglich war die Bezeichnung noch nicht mit der ,extra-terrestrial hypothesis“ (ETH, außerirdische Herkunftshypothese der UFOs) verheiratet und implizierte nur, daß etwas gesehen worden war, das mit herkömmlichen militärischen Aufklärungs- und Abfangtechnologien nicht identifizierbar schien, sich im Luftraum bewegte und physisch (solid) erschien - ein „unidentified flying object“. Dieser Begriff, für militärische Beobachter im geheimen Nachrichtenverkehr reserviert, wurde später inflationär auf alles angewandt, was „merkwürdig“, „oben“ und „räumlich begrenzt“ schien, ja sogar auf Berichte ohne Objekt und ohne Luft-Bezug (,später war es nicht mehr da - also war es weggeflogen").

Tiefenpsychologie: „Psychiatrische und psychologische Forschungsrichtung, die sich besonders mit der Bedeutung vorbewußter seelischer Gegebenheiten und den Tiefenschichten der Persönlichkeit für Seelenleben und Verhalten befaßt", sagt Peters ${ }^{7}$. Das Forschungsfeld setzt sich damit von der (meist naturwissenschaftlich orientierten) „Bewußtseinspsychologie“ ab und bezieht seelische Zustände wie Traum, Hypnose, Trance, psychotisches Erleben mit ein.

Religionswissenschaft ist ein Ausdruck für „Theologie ohne Dogma“, das heißt jene wissenschaftlich-kritische Erforschung des Heiligen, „Numinosen“ (Otto) im menschlichen Leben und in den verschiedenen Gesellschaftsformen, welche dies ohne den gleichzeitigen Anspruch "und so ist es richtig" tut.

Mythos: Frei nach Eliade eine wahre Geschichte, die von übernatürlichen Wesen erzählt, welche „vor Zeiten“ bestimmte für den heute lebenden Menschen vorbildhafte, richtige Akte gesetzt haben. ${ }^{8}$ Die symbolische Wiederholung dieser Akte ist die Basis der Ethik. Unbewußt-emotional - nicht intellektuell - hat der Mensch an der Kraft des Mythos Anteil, in einer „participation mystique“ (Levy-Bruhl). 


\section{Literaturkritik}

Eine Übersicht der erreichbaren Literatur zeigte mir bald, daß das Thema „Science Fiction - Tiefenpsychologie Religion“ trotz offiziell immer wieder heraufbeschworener „interdisziplinärer Forschung“ so gut wie ignoriert wird. Ausnahmen sind Pionierarbeiten von Sigmund Freud, Carl Gustav Jung, Margot Kadinsky (einen Überblick gibt vom Scheidt ${ }^{9}$ sowie in neuerer Zeit Wolfgang Schmidbauer ${ }^{10}$, Arnaldo Rascovsky und Mitarbeiter ${ }^{11}$, Stanislaw Grof ${ }^{12}$ und Igor Alexander Caruso. ${ }^{13}$ Um keinem eindimensionalen Psychologismus (,alles ist nur psychisch“) zu verfallen, bezog ich auch soziologisches, ethnologisches und religionswissenschaftliches Material ein.

Zu Beginn möchte ich auf die methodische Klippe eingehen, die auf den Analytiker kollektiver Phantasien lauert. Ähnlich wie die Interdependenz zwischen Kommunikator und Rezipient bei Prakke ${ }^{14}$ ist bei Massenphänomenen wie der Science Fiction eine fortlaufende dialektische Wechselwirkung zwischen individueller Psyche und Gesellschaft/ Medien anzunehmen. Für den Tiefenpsychologen Mentzos ist die psychische Entwicklung des Individuums ein „Zusammenspiel von Externalisations- und Internalisationsprozessen ${ }^{15}$ “, das heißt, es herrscht eine Art Fließgleichgewicht zwischen von „außen“" aufgenommenen und von ,innen“ kreativ nach „außen“ drängenden Bewußtseinsinhalten, wie den Gefühlen. Ein Versuch der hermeneutischen Analyse von „Science Fiction", also der Übergang von inhaltsanalytisch-deskriptiver Methodik auf ein SinnVerständnis, eine Wesensdeutung, steht damit vor dem Problem, daß die saubere Trennung zwischen Individuum und Gesellschaft zusammenbricht, so, als wären alle Individuen im „Untergrund“ (Jung: „kollektiv unbewußt") miteinander verbunden. Wie Devereux am Beispiel ethnologischer Feldforschungen nachweist ${ }^{16}$, ist auch die SubjektObjekt-Spaltung, jene aus den Naturwissenschaften importierte Trennung zwischen Beobachter und Beobachtetem, aus tiefenpsychologischer Sicht eine schöne Illusion. „Objektive“, „exakte“, „quantitativ abgesicherte“ Erkenntnis über den Menschen habe daher, wo Sinnfragen im Mittelpunkt stehen, zugunsten interaktiver, den Standort des Forschenden reflektierender, subjektiver Verfahren in den Hintergrund zu treten. ${ }^{17,18,19}$ In diesem Sinne kann der folgende Text gar nicht anders, als subjektiv-biografieverhaftet, ethnozentrisch und relativ zu sein. Ein Beitrag etwa eines Chinesen oder eines Afrikaners über „unsere Science Fiction“ wäre zu begrüßen.

Der Aufsatz „Descensus ad inferos“ von Jürgen vom Scheidt in Barmeyers Sammelband „Science Fiction" ist eine kurze, aber vollständige Darstellung der Behandlung des Themas durch die Pioniere der Tiefenpsychologie. Freud hat besonders das Gemeinsame zwischen Traum, Wahn und literarischer Phantasie, unbefriedigter, ins Unbewußte verdrängter vitaler Wünsche als Ursache der Phantasien und den im Umheimlichen wiederkehrenden Animismus (Animismus bezeichnet die Beseelung der Welt, eine narzißtische Selbstüberschätzung, die Allmacht der Gedanken, Magie) herausgestrichen. ${ }^{20,21,22,23}$ Der Lustgewinn phantastischer Dichtungen besteht nach Freud darin, „daß uns der Dichter in den Stand setzt, unsere eigenen Phantasien nunmehr ohne jeden Vorwurf und ohne Schämen zu genießen.“. ${ }^{24}$ Auf die Science Fiction- und FantasyGattung übertragen lautet also die Freudsche These, daß ihr Erfolg darauf beruht, daß sie den gesellschaftlichen Moralcodex beziehungsweise das individuelle Gewissen unterlaufen und eine symbolische Befriedigung verdrängter Triebregungen erlauben. Meiner Ansicht nach ist das im Kern richtig getroffen, aber nicht unbedingt das Spezifikum utopischer Stilrichtungen im Vergleich zu anderen Genres der populären Kultur. Sexuelle, aggressive oder „perverse“ Inhalte sind zwar unverzichtbare Ingredienzien 
utopischer Darstellungsformen, kommen aber in anderen „Ecken“ der Massenkommunikation (zum Beispiel Porno- Horror-, Gewalt-Darstellungen) unverhüllter zum Ausdruck. Wenn wir vom Freudschen Konzept der Wunscherfüllung durch Literatur ausgehen, muß zur „Vorlust“ durch symbolische Triebbefreiung noch etwas anderes hinzukommen.

Der erste Psychologe, der hier - bis zum offenen Bruch mit Freud - über den „Pansexualismus des Unbewußten" hinausgedacht hat, war Carl Gustav Jung. Während Freud mit seinem Postulat der Universalität des Ödipuskomplexes und dem Vatermord als traumatischer Grundlage für „die“ Religion ethnozentrisch verharrte, machte Jung durch Erweiterung seines seelischen Modells die Mythologie fremder Völker für die Deutung europäischer Seelenvorgänge fruchtbar. Sein „kollektiv Unbewußtes“ bildet einen gemeinsamen „Urgrund“ des Erlebens, aus dem Nichteuropäer genauso schöpfen wie wir, und die "Archetypenlehre" verbindet unsere Mythenwelt mit jener Afrikas oder Asiens durch der ganzen Menschheit gemeinsame „Ursymbole“(Archetypen) wie etwa der weise alte Mann oder die große Mutter. Bei vom Scheidt finden sich einige JungZitate in dieser Richtung. ${ }^{9}$ In der UFO-Literatur häufig zitiert und ebenso häufig mißverstanden wird Jungs Buch „Ein moderner Mythos“ aus dem Jahre $1958 .{ }^{25}$ Diese erste tiefenpsychologische Abhandlung über „Fliegende Untertassen“ weist darauf hin, daß schon Jahrhunderte vor Kenneth Arnolds Beobachtung 1947 ,Wunderdinge“ am Himmel gesehen wurden und daß bei den UFOs schon allein aufgrund der Erscheinungsform ein latenter religiöser Sinngehalt mit in Betracht zu ziehen sei, auch wenn sich ein physikalisch „reales"Substrat der Augenzeugenberichte nachweisen lasse. „Ufologen“ haben dies bis heute mehrheitlich entrüstet zurückgewiesen und befinden sich nach wie vor methodisch in der Zeit vor Jung. ${ }^{26}$

Mit der Finfuihrung des Mythos und der Archetypen in seine Betrachtungen hat Jung die von Freud unterbrochene Verbindung zwischen Tiefenpsychologie und Religion wieder hergestellt. Das Erleben archetypischer Bilder und Geschehnisse in Traum, Vision oder Tranceritual sprengt die ,gedachten“ Grenzen der eigenen Person und Kultur. Der rumänische Religionshistoriker Mircea Eliade meint im Vorwort zu „Die Sehnsucht nach dem Ursprung“: „Es ist schade, daß wir kein präziseres Wort als Religion haben, um das Erlebnis des Heiligen zu bezeichnen. Dieses Wort ist mit einer langen, kulturell ehęr eingeschränkten Geschichte belastet . ..' ${ }^{\text {(27 }}$. Einige Seiten weiter schreibt er zur angeblichen „Areligiosität“ unserer Gesellschaft: „Die Säkularisation ist äußerst erfolgreich auf dem Niveau des bewußten Lebens: alte theologische Ideen, Dogmen, Glaubenssätze, Riten und Institutionen werden immer mehr des Sinns entleert. Man kann aber keinen lebenden normalen Menschen nur auf seine bewußte rationale Tätigkeit reduzieren, denn auch der moderne Mensch träumt, verliebt sich, hört Musik, geht ins Theater, sieht Filme, liest Bücher - kurz, lebt nicht nur in einer geschichtlichen und natürlichen Welt, sondern auch in einer existentiellen, privaten Welt und in einem imaginären Universum. ${ }^{\ll 28}$

In diesem imaginären Sinn-Universum treffen sich nicht nur Tiefenpsychologen und Religionswissenschaftler (sofern nicht Dogmatismus und Ideologie zu einer „unheimlichen Begegnung der methodischen Art" führen), sondern auch Technologie und Erlösungswunsch in der Ersatzreligion des UFO-Glaubens oder keltische Sagenstoffe und Weltraumfahrt in Gestalt eines „Kriegs der Sterne“. Jung und Eliade erweitern die Freudsche Sicht der „Science Fiction/Fantasy"-Stoffe um die religiöse Dimension im „neuen“ Mythos vom Außerirdischen, von der Maschine (vgl. Anm ${ }^{8,29}$ ) und von Super- 
mann/frau lebt die längst totgesagte Götterwelt wieder auf oder, um es mit Emmanuel Geibel zu sagen: „Wenn die Götter ihr verjagt, kommen die Gespenster.“ Geschichtlicher Ursprung dieser Phantasmen einer "Wissenschaftsreligion" war nicht etwa der Atombombenabwurf, sondern, wie Michael Fischer in seiner Habilitationsschrift „Die Aufklärung und ihr Gegenteil ${ }^{\ll 30}$ und Alain Schmitt in einer noch nicht veröffentlichten Arbeit über den Mythos des Außerirdischen ${ }^{8}$ ausführen, die naturwissenschaftliche Revolution vom lebendigen Kosmos zum mechanischen Universum und die Verdammung der alten Metaphysik zugunsten einer „Objektivitätsmetaphysik“, die ihre metaphysischen Wurzeln verleugnet. ${ }^{31}$

Fassen wir zusammen: Als plausible Wirkungstheorie der „Science Fiction“ im Rahmen der modernen Massenkommunikation bieten sich symbolische Erfüllung verdrängter Triebregungen und religiöse Sinnsuche durch die Rezipienten an. Kadinsky bemerkt zu Recht, daß SF „eine ähnlich kathartische Wirkung auf ihr Publikum hat wie das Drama und speziell die Tragödie, wie der Mythos und das Märchen. ${ }^{632}$

Die Wurzeln für Typen und Handlungsabläufe in SF und Fantasy reichen sehr tief ... Biedermann leitet die UFO-Wesen und Astronautengötter unserer Zeit von den ,Astralgeistern" der antiken und mittelalterlichen Ritualmagie ab. ${ }^{33}$ Diese mit "Intelligentia“ und „Daemonium“ bezeichneten Wesen gehörten zu den fixen Bestandteilen des ,imaginären Universums“ von Aristoteles, Origines, Augustinus, Papst Innocentius VIII., Trithemius und Agrippa von Nettesheim. So hieß etwa der "gute" Planetengeist von Jupiter bei Agrippa Iophiel, der „böse“ Hismael. Die zur Anrufung der Gestirngeister nötigen Zeremonien standen nach Biedermann hinsichtlich Komplexität den modernen Instrumentarien zur Entdeckung interstellarer Radiosignale nicht viel nach. Auch „Augenzeugenberichte“, ähnlich den vielpublizierten UFO-Sichtungen, gab es damals. So sah Frater Albertus Beyer aus dem Karmeliter-Orden im Kloster Santa Maria Magdalena della Stella Nova am 2. Februar 1560 den Merkur-Geist Tiriel ,in Gestalt eines schwarzen länglichen Scheines oder Schattens", welcher ihm „mit einer hohlen Donnerstimme Rede und Antwort stand ${ }^{\star 34}$ Die Astralmagie ist jedoch nicht die einzige Verbindungslinie zwischen vorgestern und heute. Golowin gibt in „Götter der Atomzeit“ eine Reihe von Beispielen aus der Schweizer Volksüberlieferung und anderen europäischen Quellen, in denen von „Stärnelütli“ (Sternenmenschen) und ähnlichen Kontakten Himmel-Erde ${ }^{35}$ die Rede ist. Gestalten wie die Märchenfee, Baba-Jaga, Frau Holle und die „verteufelten“"Hexen tauchen in Kindermärchen und Volkssage nicht viel anders auf als Helden und Bösewichte samt ihren technologischen Hexenbesen in der „space opera“ anno 1985.

Mit der Entzauberung der Welt durch „Aufklärung “ und naturwissenschaftliche Methodik ging der gefühlsmäßige Zugang zu diesen Überlieferungen verloren. Hexen und Zauberer verschwanden im „Kinderbuch“, Okkultismus und Brauchtumsgruppen wurden zu Reservaten des austrocknenden „Phantasiens“. Berichte aus „Entwicklungsländern", deren Tradition die europäische Kolonisation überlebt hat, lassen unseren Bruch deutlich werden: Für den Schamanen, den „witch doctor“ oder „brujo“ ist die „Reise in die Oberwelt", die „Himmelfahrt“ zu den Ahnen eine erfahrungsmäßige Selbstverständlichkeit. In Harners Bericht von einer Vision unter Drogeneinfluß bei den ConiboIndianern des Amazonasdschungels findet man sich rasch in „Fantasy"-Kulissen wieder:

„Sie zeigten mir die Erde, wie sie vor Äonen, vor allem Leben war. Ich sah einen Ozean, kahles Land und einen hellen blauen Himmel. Dann fielen schwarze Punkte zu hunderten vom Himmel und landeten vor mir in der kahlen Landschaft. Es waren große, schwarze Wesen mit kleinen Flügeln, wie Flugsaurier, und walartigen 
Körpern. ... Sie landeten, völlig erschöpft von der langen Reise, und blieben lange sitzen. Sie erklärten mir in einer Art Gedankensprache, daß sie vor etwas im Weltall geflohen waren. Sie waren auf die Erde gekommen, um ihrem Feind zu entkommen."

Die Reptilien präsentieren sich Harner als „Herren der Welt". Als er einen blinden Schamanen um Rat fragt, meint dieser: „Das sagen sie immer. Sie sind aber nur die Herren der Äußeren Dunkelheit ${ }^{\star 36}$. Die phantastisch anmutende Geschichte aus der Feldforschung eines US-Anthropologen zeigt zweierlei - die Ähnlichkeit „schamanischer Visionen“ mit der SF/Fantasy-Szenerie der ,zivilisierten“ Welt und die Sinnhaftigkeit und Einordnung der Vision in ein religiös-philosophisches Weltgebäude durch den „Altmeister", der selbst genug Visionen hatte, um Harners Erlebnis kritisch hinterfragen zu können. Belege für sinnvolle Systeme in anderen fremden Kulturen finden sich unter anderem bei Joan Halifax „Die andere Wirklichkeit der Schamanen“. So führt etwa Prem Das, einen Schüler des Huichol-Schamanen Don José, seine Reise durch die „Nieríka“ (den Visionstunnel) in einen Himmel voll leuchtender Wolkenformen, hin zu einer großen leuchtenden Kugel, dem Zentrum des Alls. ${ }^{37}$

Die heftige Diskussion um das Buch „Traumzeit - Über die Grenzen zwischen Wildnis und Zivilisation“ von Hans-Peter Duerr ${ }^{\text {vgl. }} 18$ hat gezeigt, daß sich die Sympathien etablierter Wissenschaftler für das Irrationale sehr in Grenzen halten. Man fühlt sich an Immanuel Kant erinnert, der seine eigene Arbeit über die Bewohner der Planeten und ihre „Schwingungen“ später mit Druckverbot belegte ${ }^{\text {'gl. }}$. . Jung erlebte seine „Nachtmeerfahrt" (eine Zeit innerer Zerrüttung mit Visionen) nach der Trennung von Freud. Der Salzburger Psychoanalytiker Igor A. Caruso schrieb drei Jahre vor seinem Tod eine Science Fiction-Kurzgeschichte ${ }^{\text {vgl. } 13}$. „Die Wiese“ beginnt in der Salzburger Alltagswelt von Caruso, als er - gebrechlich und der Emeritierung nahe - vom Balkon seiner Wohnung aus ein UFO auf der Wiese vor dem Gebäude landen sieht. Eine Polizeiaktion mit viel Aufregung folgt. Caruso geht schlafen. In der Nacht weckt ihn ein „Alien“, der aber wieder flieht, bevor sich ein Dialog entwickeln kann. Dann verschwindet auch das UFO wieder. Caruso bleibt nachdenklich und etwas wehmütig zurück. Die versteckte Botschaft dieser Erzählung scheint mir eine ähnliche zu sein wie jene im Asteroiden-Traum von C.G. Jung, den vom Scheidt zitiert ${ }^{\text {vgl. }}{ }^{38}$.

Damit wären wir bei der entwicklungspsychologischen Bewertung der Science Fiction angelangt. Die wenigen Arbeiten zum Thema sind sich einig darin, daß SF/Fantasy stark regressive (entwicklungsgeschichtlich frühe) seelische Elemente enthält. Ob man allerdings so weit gehen sollte wie Rascovsky und Mitarbeiter ${ }^{\mathrm{vg} .11}$ oder Stanislaw Grof ${ }^{\mathrm{vgl} .12}$, die alle „überirdischen“ Phantasien auf Rückerinnerungen verschiedener vorgeburtlicher Eindrücke und Empfindungen während der Geburt reduzieren wollen, sei dahingestellt. Interessanter ist der von vom Scheidt aus der eigenen therapeutischen Praxis geschilderte Fall T.D. ${ }^{39}$. Hier trat Science Fiction in einer Entwicklungskrise als überwiegendes seelisches Ausdrucksmittel eben jener Krise auf. Die symbolischen Entsprechungen zur Lebensgeschichte von T.D. sind frappant. Mit Fortschreiten der Therapie verlieren die SF-Phantasien ihre Macht, der „alte SF-Schriftsteller stirbt“" Einen fast analogen Therapiefall beschreibt Hopf ${ }^{40}$ : Jens, ein unehelich geborener, von seiner Mutter in den ersten Lebensjahren von Pflegestelle zu Pflegestelle abgeschobener Junge, kam mit 14 1/2 Jahren nach Schulversagen mit depressiven Verstimmungen, Entfremdungsgefühlen, Arbeitsunlust und Selbstmordphantasien in Therapie. In seinen Zeichnungen, die er im Verlauf der Psychotherapie anfertigte, dominierten anfangs kalte Weltraumszenen und chaotische Raumschlachten. Auch seine Träume kreisten um Isolation und Entfremdung. Hopf beschreibt, wie sich Jens trotz starker Berührungs- 
ängste und der Angst, allein im Triebstau der Pubertät (symbolisiert durch kosmische Katastrophen) wahnsinnig zu werden, über eine „zweite Geburt“ in einer Fieberphantasie und durch Bewußtwerden seiner enormen Wut auf die Mutter (die im Traum von einem Panzer überrollt wird) langsam weiterentwickelte. Science Fiction verlor auch bei Jens als „Transportmedium“ für seine inneren Konflikte zunehmend an Bedeutung, je bewußter er seine Wünsche ausdrücken konnte.

Es wäre kurzschlüssig, mit diesen Fallgeschichten sofort Psychotherapie für SF-Leser zu fordern. Solange SF nicht zur Problemflucht, zur Aussteigerdroge wird, hat dieses Genre ebensoviel Daseinsberechtigung wie Tageszeitung und Kriminalroman. Aus der Perspektive außereuropäischer Kulturen läßt sich allerdings gegen den heute normalen SF-Konsum via Buch, Magazin, Film, Video und Fernsehen einwenden, daß hier ein kreativer, tiefenpsychologisch und religiös hochaktiver Prozeß zur Ware gemacht und damit seiner potentiellen Wirkung beraubt wird. Statt, wie es Schamanen tun, in Trance selbst „in den Weltraum“ zu fliegen, sehen wir den Helden zu, die das für uns tun und uns so unsere Entwicklung „ersparen“. Mindestens eine therapeutische Technik tut aber genau das sehr wohl: Das „Katathyme Bilderleben“" nach Hanscarl Leuner ${ }^{42}$ läßt den Patienten in einer dem Autogenen Training ähnlichen Situation innere Phantasiebilder erleben und durchwandern. „Fliegen, ... Weltraumerlebnisse, Begegnungen mit archaischen Gestalten, ... Vulkanausbruch . . . Maschinen-Imaginationen . . . Sonnenaufgang oder Sonnenuntergang . . ${ }^{43}$ und andere Motive sind für die Leuner-Schüler Indizien dafür, daß „narzißtisches“ (sehr frühes Beziehungs-) Material ins Bewußtsein steigt. Während SF-Szenerien Ausdruck einer pubertären Entwicklungshemmung sein können, werden sie im „Katathymen Bilderleben“ zum Motor der seelischen Entwicklung. Die Urbilder sind, wie alles „Numinose“44 doppeldeutig, ,ambivalent“, gut und böse. Wolfgang Schmidbauer leitet in „Unser täglicher Narzißmus“ den Typus des allmächtigen Helden tiefenpsychologisch aus narzißtischen Wunschvorstellungen her ${ }^{\text {vgl. }}{ }^{10}$.

Einen interessanten empirischen Befund stellt die Arbeit eines Teams der belgischen Universität Liège dar ${ }^{\text {vgl. }}{ }^{53}$ : Die Analyse von 15 Rorschachtests von Comic-Zeichnern (professionellen Künstlern) ergab in zwölf Fällen eine „Borderline-Persönlichkeitsstruktur", das heißt, sie lagen nach den Standards der klinischen Psychologie und Psychiatrie zwischen Neurose und Psychose, also am Rande (,borderline“) des Verlusts der Realitätskontrolle. Wenn man auch über die Deutung künstlerischer Rorschach-Antworten als „pathologisch“ oder „besonders kreativ“ streiten kann, deutet die Studie aus Lüttich in dieselbe Richtung wie die Spekulation des Autors. Comic-Zeichner sind in ihrem Persönlichkeitsgefüge weniger rigide als die übrige Bevölkerung und daher vorrangig potentielle Empfänger für symbolische Botschaften aus dem (gesellschaftlich überformten) „kollektiv Unbewußten“.

\section{Eigene Erfahrungen mit dem Thema}

Logisch gedacht, aber wer sagt, daß es wirklich so ist? - ließe sich gegen die Gedanken im Abschnitt „Literaturkritik“ einwenden: Kommt das Weltraummonster im SF-Comic deshalb vor, weil sich der Maler nach einem Nacht- oder Tagtraum mit einer ,narzißtischen Szene der Begegnung mit archaischen Gestalten“ an die Staffelei gesetzt hat? Ist der Vergleich mit Schamanenreisen nicht einfach überspannt, alternativ-ausgeflippt? Die Träume und Phantasiebilder von Jung oder Caruso erscheinen als Beleg für die Thesen etwas dürr. Gibt es Leute, die außerhalb einer psychotherapeutischen Situation SF/Fantasy-Material (möglichst nicht-kommerziell) produzieren? 
Es gibt sie zuhauf, und sie sind mir persönlich begegnet. Es handelt sich um die Beobachter „Fliegender Untertassen“, nach Rogerson ein Gebiet „partizipativer Folklore ${ }^{\text {“45, }}$ also Folklore, die laufend via Augenzeugenbericht neu im Entstehen ist. Am Beispiel der „unidentified flying objects" läßt sich gut studieren, wie ein Mythos, gesellschaftlich gebrochen in kommerziell interessante Folklore („Ufos in Salzburg gelandet"; eine McDonalds-Werbeaktion März 1985), über Massenkommunikation und Gerücht die Individuen erreicht und bei einigen in den entsprechenden lebensgeschichtlichen Situationen zu „echten“ Beobachtungen Anlaß gibt. Diese psychosoziale Seite des „UFORätsels“ wurde bis in die siebziger Jahre hinein so gut wie ignoriert. Offizielle ebenso wie private (Amateur-),,field investigators“ kamen gar nicht auf die Idee, der seelischen und gesellschaftlichen Dimension Aufmerksamkeit zu schenken. Zeugen und Untersucher verband (unbewußt) der gemeinsame Glaube, etwas wirklich Großem, einem „außerirdischen Rätsel“ auf der Spur zu sein. Hynek, Astronom und leitender UFO-Untersucher der US Air Force im Rahmen des „Project Blue Book“, beteuerte später gläubig: „The witnesses I interviewed could have been lying, could have been insane, or could have been hallucinating collectively - but I do not think so. ${ }^{\text {"46 }}$ Eine Untersuchung der Zeugen wurde gemieden wie die Pest, waren doch die Zeugen der lebende „Beweis“ für die extraterrestrische „Offenbarung“. Mit um so größerer Akribie stürzten sich Techniker auf Größen, Flugbahnen, elektromagnetische Wirkungen der „Objekte“. Bis auf einige wenige Fälle mit „harten Daten“ (Fotos, Spuren) blieb der Bericht praktisch unbekannter Zeugen der Sand, auf den fleißig gebaut wurde. In einer klassischen Bibliographie von UFO-Publikationen, zusammengestellt von Lynn Catoe in den USA, finden sich unter 1600 erfaßten Titeln nur 11 Beiträge über Wahrnehmungspsychologie - das sind 0,7 Prozent. $^{47}$

Eine erste Anregung kam vom US-Mediziner Walker. 1968 schlug er eine medizinische (neurophtalmologische, neurologische und psychiatrische) Untersuchungsmethodik für Augenzeugen vor und präsentierte gleich einen so untersuchten Fall. ${ }^{48}$ Dann wurde das Problem wieder lange verdrängt, bis Haines zwei Bücher über UFOs und Wahrnehmungspsychologie, allerdings aus der „human engineering“-Perspektive, veröffentlich$t e^{49,50} .1979 / 80$ führte ich mit Unterstützung des Wiener Kulturamtes eine psychologische Befragung von zehn Augenzeugen naturwissenschaftlich nicht erklärbarer Himmelserscheinungen im Raum Wien durch. ${ }^{51}$ Nach anfänglichen Schwierigkeiten mit der Fallbeurteilung - ein klinischer Psychologe, der als Senior die Test-Auswertung fertigstellte, sah so gut wie jeden Beobachter als „Neurotiker" an - zeigte sich bei weiteren Fallstudien, daß ein tiefenpsychologischer Test (der Rorschach-Test) und eine kurze Aufnahme der Lebensgeschichte oft Teile der Beobachtungen in ganz anderem Licht erscheinen ließen, und daß die Beobachter nicht, wie ursprünglich vermutet, psychopathologischen Phänomenen aufgesessen waren, sondern normale Menschen waren, die „Abnormales“ berichteten.

Während eines Englandaufenthaltes 1981 besuchte ich mit einem Freund dort Beobachter ungewöhnlicher Himmelsphänomene und konnte dieselben Strukturen feststellen wie in Österreich. Eine Publikation darüber ist in Vorbereitung.

Als typische Beispiele für „partizipative Folklore“, beziehungsweise einen „lebendigen Mythos“, möchte ich zwei Fälle stark verkürzt anführen. 


\section{Zwei Fallbeispiele}

Um den Durchbruch unbewußter Phantasien in Form von ,realen Beobachtungen“ $z u$ illustrieren, gebe ich je ein knapp dargestelltes Fallbeispiel aus Österreich und Großbritannien an.

\section{1 Österreich}

Eine 24jährige Kosmetikerin, wohnhaft in einer Ortschaft an der Donau nordwestlich von Wien, rief die Sternwarte an und gab an, sie hätte mit ihrem Freund am 31. Oktober 1980 gegen vier Uhr morgens zwei helle Lichter tief am Himmel hängen sehen. Sie fuhr dann mit ihrem Auto allein auf einen Parkplatz an der Donau und stieg aus. Die Lichter am Himmel sahen wie große Autoscheinwerfer aus, bewegten sich nicht und schienen sie an. Nach etwa einer halben Stunde an der einsamen Stelle sei es ihr unheimlich geworden und sie fuhr heim, wo sie bemerkte, daß ihr Gesicht und die Augen entzündet (gerötet) waren. Sie beschrieb Symptome eines leichten Sonnenbrandes und einer Bindehautentzündung (Conjunctivitis). Die Symptome verschwanden während des Tages.

Als ich wegen meiner Felduntersuchung rückrief, fiel mir auf, daß die Mutter der Kosmetikerin (etwa 40 Jahre alt), eine Künstlerin und Schriftstellerin, die treibende Kraft hinter dem Erstbericht war. Die Zeugin war eher zurückhaltend, ihre Mutter fast überschwenglich interessiert und gerne bereit, ihre Tochter für einen Untersuchungstermin zu gewinnen. De facto (wie sich später herausstellte) zwang sie die Tochter zu erscheinen, was sich bei jener in unwirschem Auftreten äußerte. Bei einem Interview und einem „Lokalaugenschein“ am 21. Dezember 1980 nahmen wir einen Rorschach-Formdeuteversuch mit der Tochter auf, der auf einigen Tafeln die Auffälligkeit zeigte, daß sie sich „angeblickt“ fühlte. Ihre Mutter gab zu den Tafeln lebhafte, gefühlslabile Deutungen ab. Eine Erklärung der beobachteten Objekte als Flugverkehr schied aus - er begann am fraglichen Tag erst um fünf Uhr früh. Allerdings standen die hellen Planeten Venus und Jupiter in Konjunktion (Nahestellung, etwa einen Monddurchmesser nahe) tief im Osten. Eine traumatische oder infektiöse Bindehautentzündung klingt unter Therapie erst nach zwei oder drei Tagen ab. Das rasche Abklingen der Symptome sprach für eine psychosomatische („hysterische“) Entstehung. Interessant waren die Geschichten, die die Mutter „so nebenbei“ erzählte: Sie war lebhaft an UFOs interessiert und überzeugt, auch „außersinnliche Wahrnehmungen“, unter anderem Vorahnungen, gemacht zu haben. Ein Freund von ihr hätte nachts UFO-Wesen aus einem Wandspiegel kommen gesehen, sie korrespondierte mit einem Professor in Campinas, Brasilien, der selbst UFOs gesehen hatte, und „wußte genau“, daß jenes Haus, in dem ihre Tochter jetzt lebte, schon Ort von UFO-Landungen gewesen war und (so eine bereits verstorbene alte Frau) daher „ein guter Platz" sei. Die Tochter, erst eher unwirsch, weil zur Teilnahme gezwungen, nahm gegen Ende der Untersuchung auch am Gespräch teil. Sie erzählte, daß sie einmal einen funkelnden Stern von ihrem Wohnungsfenster aus gesehen habe, der ihr eine „Botschaft“ zublinkte. Sie war sicher, daß die beiden Begegnungen nur „ein Vorspiel zu einem großen Kontakt" gewesen seien.

Wir schlossen die Untersuchung mit der starken Vermutung ab, daß die „Beobachtung“ eine „hysterische“ (psychosomatische Symptome bildende) Delegation war, also die Tochter gegen ihren bewußten (intellektuellen) Widerstand die unbewußten Phantasien der Mutter auslebte. Weitere Beobachter meldeten sich nicht. Eine mögliche Erklärung für die „Scheinwerfer“ des „UFOs“ wären die zwei tiefstehenden Planeten, die der Tochter in einem veränderten Bewußtseinszustand so groß erschienen und auch in der 
entsprechenden Himmelsgegend standen. Ein Anhänger der „außerirdischen Herkunft“ der „UFOs“ (extraterrestrische Hypothese, kurz E.T.H.) hätte die Daten allerdings anders gedeutet.

\subsection{Großbritannien}

Ein 27jähriger, seit 1981 arbeitsloser Nachtwächter, der mit einer 56 Jahre alten, ebenfalls arbeitslosen Sekretärin und deren 16jährigem Sohn in einer Wohnung einer Großstadt westlich von London zusammenlebt, wandte sich 1984 an eine private UFOUntersuchungsgruppe. Sein schriftlicher Bericht wurde von einem lokalen Untersucher rasch an meinen englischen Freund weitergereicht. Am 20. Februar 1985 sprachen wir beide einen Nachmittag lang mit der Familie. Einem vierseitigen, maschinengeschriebenen Bericht des Zeugen und seinen persönlichen Erklärungen konnten wir entnehmen, daß er seine Frau 1979 kennengelernt hatte (sie lebt in Scheidung von ihrem ersten Mann, einem Polizeioffizier) und sie seit 1981/82 das Gefühl hatten, mit übernatürlichen Kräften in Kontakt zu kommen. Aus dem reichen Spektrum der nun folgenden Manifestationen seien nun zwei als wörtliche Übersetzung aus dem Zeugenbericht angeführt:

(Eine Beobachtung der Frau) ,... in einem Teil von (Stadt), der (Name) heißt, einer offenen Fläche nahe der (Stadt)-Universität. Eines Abends ging sie mit ihren Hunden über dieses Terrain, als sie sich zu einer Baumgruppe hingezogen fühlte und bei der Annäherung das Erlebnis hatte, eine ,Barriere aus unsichtbaren Spinnweben' zu durchschreiten. Sie verlor die Orientierung und sah sich mehreren zweibeinigen Reptilwesen gegenüber, die dann eine Art medizinischer Untersuchung in einem Laboratorium mit ihr durchführten. Um dies später herauszufinden, mußte ich sie (meine Frau - d.Ü.) hypnotisch rückführen, da sie bewußt nichts mehr an den Vorfall erinnerte . . . aber mehrere Stunden Zeit verloren hatte bei einem Spaziergang, der normalerweise ein bis eineinhalb Stunden hätte dauern können.“

„Das letzte Ereignis war in der Nacht des 10. Dezember 1984 und setzt mich noch immer in Erstaunen. Ich schlief, als ich plötzlich gewahr wurde, daß mein Körper vibrierte ... In meinen Ohren war ein lautes, sausendes Geräusch wie Wind oder Wasser. $\mathrm{Zu}$ diesem Zeitpunkt geriet ich ohne Grund (? - d.Ü.) in Panik und kämpfte mich in einen halbwachen Zustand hoch. Schließlich war ich wach und rüttelte (meine Frau - d.Ü.), aber sie erwachte nicht, auch nicht, als ich sie an den Haaren riß. Ich richtete mich dann auf, um nachzusehen, warum sie nicht aufwachen wollte, und war entsetzt, ein strahlendes Licht als Reflex auf ihrem Gesicht zu sehen, welches ich auszustrahlen schien. Dann hörte ich einen Schrei, ein Lichtball kam aus meinem Körper und fuhr in die Mitte des Zimmers, wuchs bis etwa vier Fuß Durchmesser (über einen Meter - d.Ü.) an und blieb im Flur am Boden sitzen, wobei er weiter ins Zimmer schien. Ich fühlte, wie mich eine Hand wieder zum Niederlegen zwang ... die Vibrationen dauerten noch einige Momente an. Dann wachte ich voll auf und auch (meine Frau - d.Ü.) - mein Puls und meine Atmung waren sehr hastig. . . . Am folgenden Tag fühlte sich (meine Frau - d. $\ddot{U}$.) ruhelos und deprimiert und sie beklagte sich, daß sich ihr Kopf in der Augen- und Ohrregion seltsam anfühle - das war die rechte Kopfseite, die Seite, auf die das Licht, welches ich ausgestrahlt hatte, gefallen war."

Die weiteren Phänomene waren zahlreich und den geschilderten ähnlich - eine Attacke auf den Zeugen durch einen „unsichtbaren Dolch“, seine Veränderung in ein mühsam atmendes Reptil (dem Körpergefühl nach), „Kontakt" mit einem Ritter des Templerordens, der Angriff einer Riesenspinne aus Atlantis, ein zwei Meter hohes Reptil, das hinter dem Wagen der Zeugen nachts über die Straße hüpft, ein im Zimmer wie im 
Wasser herumschwimmender Fisch, ein Riesenmoskito, ein großer haariger Affe neben dem Schreibmaschinentisch. Die Reptile erinnern an die Vision von Harner. Für den Zeugen, der inzwischen auf Anordnung eines außerirdischen „Wissenschaftlers“ - übrigens ein schuppiges, echsenartiges Wesen - ein Buch zu schreiben begann, verfolgten die Manifestationen den Zweck, sie beide mit dem Wissen einer „galaktischen Strafkolonie nahe Capella“ bekanntzumachen, die aus Wesen von Atlantis besteht, welche ihre Gehirne in Reptilkörper verpflanzt haben. Dieses Wissen wäre für die Erde sehr wichtig, weil ,ab den neunziger Jahren keine Zukunft für das weitere Leben auf der Erde" bestünde und große Katastrophen bevorstünden. Eine millenistische Kundgabe also.

Die Frau des Zeugen berichtet schon aus der Zeit vor dem „außerirdischen Kontakt“ von ungewöhnlichen Fähigkeiten - sie erlebte „Reisen“ außerhalb ihres Körpers, sah Feen, konnte heilen, hatte präkognitive Träume, konnte hellsehen und so weiter. Sie hält sich nicht für religiös. Der Zeuge kam erst seit Beginn der Partnerschaft mit der 29 Jahre älteren Frau mit solchen Fähigkeiten in Kontakt - derzeit betreibt er „Astralreisen“, Telepathie und schreibt an seinem vom Reptil „Wissenschaftler" diktierten Buch.

Rorschach-Formdeutversuche mit den beiden Personen erbrachten bei der Zeugin keine besonderen Details; der Zeuge hingegen schien etwas paranoid beziehungsweise sozial überängstlich zu sein. Die Inhalte der Wahrnehmungen stammen aus dem Repertoire der okkulten Tradition, der christlichen „Dämonologie“ und aus dem Märchen/ Sagen- und Fantasy-Bereich. Der Zeuge hat nach eigenen Angaben eine Menge grenzwissenschaftlicher Literatur gelesen. Aus seinem Bericht wird deutlich, daß er die Visionen in Zuständen veränderten Bewußtseins (vor allem nachts) hatte. Da der Zeuge einer religiösen Einordnung des Gesehenen skeptisch gegenüberstand, gab ich ihm die Adresse der „Spiritualist Association of Great Britain“ für eventuelle Beratung und Hilfe.

\section{Resultate}

Die wenigen Literatur- und Fallbeispiele sollten skizzenartig einen Eindruck von unbewußten Kraftflüssen vermitteln, die für die Struktur des ,imaginären Universums“ (Eliade) in unserer Zivilisation von Bedeutung sind. Durch UFO-Spiele und -Filme, Fantasywesen im Kinderzimmer (besonders die Serie „Masters of the Universe“) und entsprechende Werbeinhalte erfährt der heute Heranwachsende eine Konditionierung auf Symbole, die in veränderten Bewußtseinszuständen (Traum, Rausch, Vision) - kreativ abgewandelt und auf die persönliche Lebensgeschichte bezogen - aus dem Unbewußten auftauchen können und eventuell dem Mythos vom Außerirdischen durch „echte Beobachtungen“ neue Nahrung geben. Bestehende gesellschaftliche Defizite körperfeindliche, gefühlsarme Erziehung, Säkularisation, Sinnmangel - erhalten so, entsprechend der Freudschen Regel vom „Wiederholungszwang“ beziehungsweise von der „Wiederkehr des Verdrängten“ die Chance, auf phantastische Weise symbolisch ins Bewußtsein zu treten und Aufmerksamkeit auf sich zu ziehen. Ein willkürlich herausgegriffenes Beispiel dafür ist etwa ein Bild des französischen SF-Künstlers Philippe Caza ${ }^{52}$ : Ein dunkler, haarloser, durch rote Schlitzaugen dämonisch wirkender nackter Frauenkörper zwischen männlich-aggressiven, Blitze werfenden, spitzen Raumschiffen vor dunklen Planetenkugeln stellt, galaktisch verfremdet, die Polarität männlich-weiblich und die destruktive Seite der Körperlichkeit dar. Die Kombination UFOs/Fantasy und Triebbereich/Archetypen kommt so häufig vor, daß von Zufall keine Rede mehr sein 
kann. Derartige „außerirdische Rorschachbilder“ machen dem griechischen Wortsinn von Symbol, nämlich „symballein“ = zusammenwerfen alle Ehre, indem sie bewußte und unbewußte Phänomene wie in einem Brennglas sammeln. Lust, Angst, Aggression, Sinnwunsch, Mystik, Ästhetik beginnen aus ein paar farbigen Linien zu wuchern.

Ich glaube, meine seinerzeit im Seminar geäußerte These, SF/Fantasy sei eine symbolische Darstellung unbewußter Triebkonflikte und religiöser Inhalte in technisch-wissenschaftlichem Gewand, konnte durch das hier kurz dargestellte Material soweit erhärtet werden, daß sie als Arbeitshypothese brauchbar erscheint. Die in jüngster Zeit in den Medien referierte Kritik am Freudschen Lehrgebäude trifft in erster Linie die Dogmatik der Psychoanalyse, ihre Sexualmythologie (mit einem englischen Scherzwort: „Everything is ,cave or' vex and therefore has to do with sex") bei gleichzeitiger Anlehnung an die Religion. Jungs System ist demgegenüber offener, weniger reduktionistisch und eignet sich gut für die Analyse von Science Fiction. Im kasuistischen Teil schließlich sollte gezeigt werden, daß schöpferische Menschen - ob SF Comic-Zeichner oder UFO-Zeugen spielt eigentlich keine Rolle - in veränderten Bewußtseinszuständen sehr lebhafte Eindrücke des ,imaginären Universums“ (Eliade) erhalten, die durch Interdependenz zwischen den „Visionären“ und den Rezipienten ihrer Botschaften zu einer laufenden Neuschöpfung des Mythos vom E.T. führen.

\section{Anmerkungen}

1 Angriff auf das Reich des König Ödipus. In: Der Spiegel, 52/1984 (24.12.), 116-132

2 E. Barmeyer (Hrsg.): Science Fiction. München: Fink 1972, 7

3 E. Barmeyer, a.a.O., 9

4 L. Wanke: Felsbilder und Traumbewußtsein. Österr. Hochschulzeitung, März 1984, I

5 E. Barmeyer, a.a.O., 11

6 H. Nagl: Science Fiction. Narr: Tübingen 1981, 114

7 U.H. Peters: Wörterbuch der Tiefenpsychologie, Geist und Psyche, München: Kindler 1978, 152

8 A. Schmitt: Der Mythos des Außerirdischen. Unveröff. Man., Wien 1984

$9 \mathrm{~J}$. vom Scheidt: Descensus ad inferos. In: E. Barmeyer (Hrsg.): Science Fiction. München: Fink $1972,133-162$

10 W. Schmidbauer: Die Ohnmacht des Helden. Reinbek/Hamburg: Rowohlt 1984

11 A. Rascovsky (Hrsg.): Die vorgeburtliche Entwicklung. Geist und Psyche. München: Kindler 1978

12 St. Grof: Topografie des Unbewußten. Stuttgart: Klett-Cotta 1983

13 I.A. Caruso: Die Wiese. In: Die Außerirdischen sind da. München: Matthes \& Seitz 1979

14 H. Prakke et al.: Kommunikation der Gesellschaft. Münster: Regensberg 1968, $64 \mathrm{f}$.

15 St. Mentzos: Neurotische Konfliktverarbeitung. Geist \& Psyche. München: Kindler 1982, 51

16 G. Devereux: Angst und Methode in den Verhaltenswissenschaften. Frankfurt Berlin Wien: Ullstein 1976

17 W.J. Revers: Ideologische Horizonte der Psychologie. München: Pustet 1962

18 H.P. Duerr: Traumzeit. Syndikat. Frankfurt/Main 1983

19 H.P. Duerr (Hrsg.): Der Wissenschaftler und das Irrationale, Bd. 1 und 2. Frankfurt/Main: Syndikat 1981

20 S. Freud: Die Traumdeutung. Frankfurt/Main: S. Fischer 1968

21 S. Freud: Der Wahn und die Träume in W. Jensens, Gradiva'. Ges. Werke VII. Frankfurt/Main:

S. Fischer 1966, 29-125

22 S. Freud: Der Dichter und das Phantasieren. Ges. Werke VII. Frankfurt/Main: S. Fischer 1966, 211-232 
23 S. Freud: Das Unheimliche. Ges. Werke XII. Frankfurt/Main: S. Fischer 1966, 227-268

24 S. Freud, a.a.O., 223

25 C.G. Jung: Ein moderner Mythus. Zürich: Rascher 1958

26 Int. UPIAR Colloquium on Human Sciences and UFO Phenomena, Salzburg July 26-29, 1982, Proceedings, UPIAR, Milano 1983

27 M. Eliade: Die Sehnsucht nach dem Ursprung. Wien: Europaverlag 1973, 9

$28 M$. Eliade, a.a.O., $12 \mathrm{f}$.

29 D. Kadinsky: Der Mythos der Maschine. Bern 1969

30 M. Fischer: Die Aufklärung und ihr Gegenteil. Berlin: Duncker \& Humblot 1982

31 W.J. Revers: Ideologische Horizonte der Psychologie. München: Pustet 1962

32 D. Kadinsky: a.a.O., 231

33 H. Biedermann: Wunderwesen Wunderwelten. Graz: Verlag f. Sammler 1980, 95 ff.

34 H. Biedermann, a.a.O., 104

35 S. Golowin: Götter der Atom-Zeit. Bern: Morzsinay 1979, 124-127

36 M. Harner: The Way of the Shaman. Toronto/New York/London/Sydney: Bantam 1982, 5-9

37 J. Halifax: Die andere Wirklichkeit der Schamanen. Berlin: Goldmann 1985, 290

$38 \mathrm{~J}$. vom Scheidt, a.a.O., 142 f.

$39 \mathrm{~J}$. vom Scheidt, a.a.O., 145-153

40 H.H. Hopf: Kinderträume. Reinbek/Hamburg: Rowohlt 1980, 58-70

41 H.H. Hopf, a.a.O., 62

42 H. Leuner: Katathymes Bilderleben. Stuttgart: Thieme 1970

$43 \mathrm{~J}$. Pahl: Über narzißtische Entwicklungslinien während des Katathymen Bilderlebens. In: H. Leuner (Hrsg.): Katathymes Bilderleben. Ergebnisse in Theorie und Praxis. Wien: Huber $1980,99 \mathrm{f}$.

44 R. Otto: Das Heilige. München: C.H. Beck 1963

45 P. Rogerson: Saucers \& Broomsticks. In: MAGONIA 18, 1/1985 (London), 10

46 J.A. Hynek: The Hynek UFO Report. New York: Dell 1977

47 F. Izzo: The Scandal is $0,7 \%$ In: Int. UPIAR Colloquium on Human Sciences and UFO Phenomena, Salzburg, July 26-29, 1982, Proceedings, UPIAR, Milano 1983, 7

48 S. Walker, in: Hearings Comm. Sc. Astron., 90th Congress, Washington D.C. 1968

49 R.F Haines (Ed.): UFO Phenomena and the Behavioral Scientist. Metuchen: Scarecrow Press N.J. 1979

50 R.F Haines: Observing UFOs. Chicago: Nelson-Hall 1980

51 A.G. Keul: Persönlichkeitsvariable und Realitätsprüfung ungewöhnlicher Zeugenaussagen. Privatdruck. Wien 1980

52 Ph. Caza: Gesammelte Werke beim Volksverlag, Bd. 3. Linden: Cover, Volksverlag 1980

53 M. Timsit et al.: Test de Rorschach avec épreuve graphique chez seize dessinateurs-illustrateurs.

In: Psychologie Française, 1983 (Jun), Vol. 28(2), 164-180 


\section{SUMMARY}

In this paper, science-fiction and the fantasy genre are approached in an inter-disciplinary, but mainly psychological and anthropological angle. Besides popular views on similar phenomena are quoted, as can be found in ethnographic reports, in psychoanalysts' and their patient's fantasies, and in the "UFO-visions" of two eye witnesses from Austria and Great Britain. These different realms are interpreted as alternative concepts invading the subconscious. Following C.G. Jungs concept of the „,collective unconscious“, modern science-fiction is therefore only a new symbolism for a traditionally-known phenomenon. The author assumes that the appearance of irrational materials from the outer cosmos yields on the one hand to a cultural specific surpression of the physical body, and on the other hand to religious needs, repressed by secularisation. Science-fiction and fantasy, the author concludes, are expressions of this interdependency (cf. Prakkes terms for the inter-relations in the process of mass communications).

\section{RÉSUMÉ}

Cet article mêle science-fiction et fantastique, quoi que d'un point de vue psychologique et anthropologique. En outre, il traite des vues diffusées sur des phénomènes parallèles que l'on peut trouver dans des rapports ethnographiques, dans les fantaisies des psychanalystes et de leurs patients ou dans les visions d'OVNI par deux témoins occulaires en Autriche et en Grande Bretagne. Ces secteurs du monde de l'étrange sont présentés comme différents chemins pour pénétrer l'inconscient. Par conséquent, la science-fiction moderne n'est - à l'instar de „'inconscient collectif" de C.G. Jung - qu'une symbolique nouvelle d'un phénomène très ancien. Lauteur suppose que l'apparition d'objets irrationnels venus de l'espace procèdent d'une part du refoulement culturel des besoins primaires et d'autre part des besoins religieux refoulés par la sécularisation. Selon l'auteur, la science-fiction et le fantastique sont une expression de cette interdépendance (cfr. la terminologie de Prakke pour parler des relations de tous les facteurs dans le processus de la communication de masse).

\section{RESUMEN}

En este artículo se mezcla la ciencia-ficción y lo fantástico, pero desde un punto de vista sicológico y antropológico. Además se refiere a las opiniones que se han difundido sobre los fenómenos paralelos y que se pueden encontrar en los informes etnográficos, en las fantasias de los sicoanalistas y de sus pacientes o en las visiones de OVNIS de dos testigos oculares en Austria y en Gran Bretaña. Estos sectores del mundo de lo extraño son presentados como diferentes caminos para penetrar el inconciente. En consecuencia la ciencia-ficción moderna no es más que el nuevo simbolismo de un fenómeno muy antiguo, el reflejo del „inconciente colectivo“ como lo ha calificado C.G. Jung. El autor spone que la aparición de objetos irracionales desde el Espacio provienen por una parte del rechazo cultural de necesidades primarias y por otra parte de necesidades religiosas abandonadas por causa de la secularisación. Según el autor, la ciencia-ficción y lo fantástico son una expresión de esta inter-dependencia (cfr. la terminología de Prakke para hablar de las relaciones de todos los factores en el proceso de comunicación de masas). 